Erschienen in: Stickel, Gerhard (Hrsg.): Varietäten des Deutschen. Regional- und Umgangssprachen. - Berlin, New York: de Gruyter, 1997. S. 88-108. (Institut für deutsche Sprache. Jahrbuch 1996)

DIETER STELLMACHER

\title{
Sprachsituation in Norddeutschland
}

\begin{abstract}
Der nicht selten als dialektfrei geltende norddeutsche Raum erweist sich in Wahrheit als ein kompliziertes Mehrsprachenland. Neben der in der Regel gut beherrschten Standardsprache finden sich die dem Standard gegenüber deutlich unterschiedenen Abstandsprachen Niederdeutsch und Friesisch sowie das Dānische. Ihre Statusproblematik, die geographische Verteilung und die Kommunikativităt der die norddeutsche Sprachsituation bildenden Sprachen werden erläutert, wobei auch das kulturelle Umfeld, in dem sich das Sprachennebeneinander entfaltet, beschrieben wird. Der Schwerpunkt der Ausführungen liegt auf dem Niederdeutschen, für dessen vielfălige Verwendung Textbeispiele angeführt werden.
\end{abstract}

Frage: "Is Plattdütsch 'ne Sprak oder bloß 'ne Mundort?" Antwort: "Plattdütsch is 'ne Sprak. Plattdütsch is 'ne Sprak, de väle Mundorden hett, so wür' ik seggen" (Herrmann-Winter 1989, S. 39). Mit diesen Worten beantwortet ein 1928 in Rostock geborener Kunstprofessor die "Grundfrage" der niederdeutschen Sprachwissenschaft, und er steht mit seiner Meinung nicht allein. Die Auffassung von der Sprachlichkeit des Plattdeutschen und damit verbunden die Abweisung seines Dialektstatus ist ein Kennzeichen norddeutscher Sprachsituation. Es wird verstärkt durch das verbreitete Wissen um den linguistischen Abstand aller norddeutschen Sprachformen zur Standardsprache (mit Ausnahme der mitteldeutschen Sprachinsel im Westharz). Wenn die Abstandsprachen Niederdeutsch und Friesisch dennoch auch als Dialekte bezeichnet werden, dann allein in einem soziolinguistischen Sinn, der sich auf die aktuellen Verwendungsweisen dieser typologisch eigenständigen Sprachen bezieht. Die Soziolinguistik unterscheidet gegenwärtig zunehmend neben der Standardsprache und den Dialekten noch Minderheitensprachen und plaziert sie zwischen Standard und Dialekt. Damit wird einer sprachgeschichtlichen Realität Rechnung getragen und diese in einem etwas martialischen Bild wiedergegeben: Sprache ist ein Dialekt mit einer Armee; Dialekt ist eine Sprache ohne Armee; Minderheitensprache ist eine Sprache, die einmal eine Armee hatte (Feitsma 1995, S. 885). Die sich aus diesen Überlegungen ergebenden Besonderheiten Norddeutschlands sind mir AnlaB, von den üblichen Vortragsformulierungen des Tagungsblocks ("Sprachvarietäten in ...") abzuweichen und meine Ausführungen unter das Thema "Sprachsituation in Norddeutschland" zu stellen. Sie 
ist geprägt durch eine vitale Mehrsprachigkeit, die nicht selten verkannt oder unterschätzt wird, z.B. wenn man unseren Raum als dialektfrei bezeichnet, oder wenn - wie in einem aktuellen Heft des ${ }_{n}$ Sprachdienstes” nachzulesen - dem Friesischen und dem Niederdeutschen der baldige Sprachtod vorhergesagt wird. ${ }^{1}$

Ich erläutere erstens, was ich unter "Sprachsituation” verstehe (1), beschreibe zweitens die an ihr beteiligten Sprachen (2), bestimme in einem dritten Abschnitt ihr Verhältnis zueinander (3), gehe viertens auf das kulturelle Umfeld ein (4) und schließe mit einer kurzen Zusammenfassung (5).

\section{Die Sprachsituation}

Der aus der sowjetischen Soziolinguistik stammende Terminus, russisch jazykovaja situacija (Vinogradov 1990, S. 616f.), wird in der Sprachwissenschaft häufig benutzt, aber kaum definiert. ${ }^{2}$ Dafür scheint seine Durchsichtigkeit verantwortlich zu sein. Die allgemeinste Bestimmung von Sprachsituation ist der Bezug auf die „Gesamtheit der Existenzformen (sowie Stilformen) einer Sprache oder einer Gesamtheit von Sprachen in ihrer territorialen und sozialen Wechselbeziehung in den Grenzen bestimmter geographischer Regionen oder administrativ-politischer Gebilde" (Vinogradov 1990, S. 616, aus dem Russischen übersetzt). Im besonderen wird die Sprachsituation durch quantitative und qualitative Merkmale bestimmt. Zu den quantitativen Merkmalen gehören die Zahl der beteiligten Sprachen (ein- oder mehrsprachige Situation), die Anzahl der Sprachträger und der von den Sprachen bedienten Kommunikationsbereiche. Zu den qualitativen Merkmalen zählen das Verwandtschaftsverhältnis der Sprachen (genetisch-strukturell ähnlich - unähnlich), ihre Status (gleichwertig - ungleichwertig) und ihre Verwendungsfähigkeiten (unbeschränkt - beschränkt). AuBerdem treten noch subjektive Bewertungsmerkmale hinzu, die für die Sprachloyalität verantwortlich sind.

Die norddeutsche Sprachsituation ist geprägt durch eine Mehrsprachigkeit „unähnlicher” Sprachen mit „ungleichwertigem” Status, aber prinzipiell unbeschränktem Verwendungsumfang. Für ihre Bewertung spielt das kulturelle Umfeld eine ausschlaggebende Rolle.

1 Sprachdienst 6 (1995), S. 202.

2 Aber Scharnhorst (1978); Semenjuk (1985, bes. S. 1451). 


\section{Die Sprachen}

Was als Norddeutschland gilt, soll sprachgeographisch bestimmt werden, und zwar als der nördlich der Ürdinger Linie gelegene Teil Deutschlands. Die auffalligste dialektale Sprachform ist hier das Niederdeutsche. ${ }^{3}$ Nicht behandeln werde ich die norddeutschen Umgangssprachen, weil sie Aktionsformen der regional markierten Standardsprache sind und nicht als ${ }_{n} Z$ weitsprache" des Norddeutschen gelten können."

Nicht behandeln werde ich ihrer speziellen Problematik wegen Sondersprachen. Schließlich behandle ich auch nicht die Sprachen von in Norddeutschland lebenden Ausländern (ihr Anteil an der Wohnbevölkerung in den "niederdeutschen Kernländern" Schleswig-Holstein, Niedersachsen und Mecklenburg-Vorpommern beträgt $4,08 \%)^{5}$, weil dieses Thema meinen Beitrag überfordern würde.

Das Niederdeutsche verfügt als Folge beharrlicher Ausbaubemühungen über standardähnliche Formen, die Dialekten sonst nicht eigen sind. Bei den beiden friesischen Varietäten, dem Saterfriesischen und dem Nordfriesischen, sind gleichfalls erfolgreiche Ansätze zur Überwindung des Dialektstatus zu beobachten, im Saterfriesischen schwächer als im Nordfriesischen, das sich derzeit sogar mehrere "Schulsprachen" leistet (was wiederum ein Beweis der Relativität dieses Sprachausbaus ist).

Entscheidend ist für das Friesische und das Niederdeutsche, daB beide Sprachen keine ausschlieBlichen Verwendungsweisen haben, in jedem $\mathrm{Be}$ reich untereinander oder mit der Standardsprache konkurrieren. Das hat vor allem im „Mehrsprachenland Schleswig-Holstein” ein kompliziertes Sprachennebeneinander zur Folge. Im schleswigschen Landesteil gibt es an der Westküste und den davor gelegenen Inseln das Festlandnordfriesische (von Ockholm bis Niebüll) und das Inselnordfriesische (auf Helgoland, Sylt, Föhr, Amrum). In Grenznähe (genauer in dem grenznahen Dreieck Flensburg-Bredstedt-Süderlügum) ist bei der dänischen Minderheit das Reichsdänische verbreitet, im Abschnitt Flensburg-LeckSüderlügum die südjütische Mundart, das Plattdänische. ${ }^{6}$

3 Wer das Niederdeutsche bei einer Darstellung der „Sprachen Europas” übergeht, zieht sich unweigerlich Kritik aus dem kulturellen Umfeld $z$, vgl. Dieter Bellmanns Rezension von Harald Haarmann, Soziologie und Politik der Sprachen Europas (1975), in: Quickborn 66 (1976), S. $63 f$.

4 Kettner (1988). Zu den Verhälnissen im ostniederdeutschen Sprachraum siehe Schönfeld (1986).

5 Stand vom 31.12.1994 laut "Welt am Sonntag” vom 10.3.1996, S. 29.

6 Vgl. Hansen/Johannesen/Runge/Steensen (1993). 
Das Saterfriesische, ein Ostfriesisch, hält sich in einigen ehemals wenig zugänglichen Ortschaften im Nordwesten das Landkreises Cloppenburg ("Oldenburger Saterland").

Eine Ahnung von der Vielgestaltigkeit der norddeutschen Sprachvarietäten zeigt die anliegende Karte. In 34 Orten habe ich nach dem 6. Wenkersatz vier Variablen kartiert: die monophthongische oder diphtongische Entwicklung von mnd. $\hat{e}^{2}$ (hêt 'heiB') (siehe DSA-Karte 16) und $\hat{o}^{1}$ (Kôken 'Kuchen') (siehe SA-Karte Kuch[en] geordnet nach dem mhd. Stammsilbenvokalismus), die palatale $\left[\int v\right]$ oder alveolare [sv] Aussprache des konsonantischen sw-Anlauts ( "schwarz") und die Präteritumform war. Die hier nur angedeutete Arealität vermittelt auch einen Eindruck von den Substanzunterschieden. Aber wichtiger als das ist für die Skizzierung der norddeutschen Sprachsituation die Kommunikativität der betreffenden Sprachen, hängt doch daran ihre Zukunft. Voraussetzung für den kommunikativen Einsatz von Sprachen ist ihre Beherrschung bei einer genügend großen Zahl von Sprechern, so daß ausreichende Gesprächspartner vorhanden sind. Nun gibt es hinsichtlich der Sprachfähigkeiten in Norddeutschland und darüber hinaus Aussagen, die fast Allgemeingültigkeit beanspruchen. Danach sind es ältere Leute aus dem ländlichen Milieu mit niedrigerem SchulabschluB und oft männlichen Geschlechts, die den idealen Sprecher des Niederdeutschen und des Friesischen abgeben. ${ }^{7}$ Das mit Zahlen zu belegen, wäre nicht sehr spannend und würde anderes, Kennzeichnenderes zu kurz kommen lassen. Dazu gehören die dialektstarken und dialektschwachen Gebiete. Zunächst zum Niederdeutschen. Stark bedrängt und teilweise bereits verdrängt ist das Niederdeutsche im Märkisch-Brandenburgischen (den Kreisen Potsdam und Oranienburg), wo die alte mittelmärkische Mundart kaum noch anzutreffen ist. ${ }^{8}$ "Allgemeines Verständigungsmittel im täglichen mündlichen Verkehr ist nicht mehr die Mundart, sondern eine überregionale, weitgehend vom Berlinischen geprägte Umgangssprache" (Wiese 1993,

7 Dem ist vergleichbar, was in der niederländischen Dialektologie als N.O.R.M. bezeichnet wird: Niet mobiele, Oudere Rurale Mannen, siehe Hoppenbrouwers, C. (1990).

8 „Mitarbeiter des BBW konnten ... bei Mundartaufnahmen in den Jahren 1960/61 in den Berlin unmittelbar benachbarten Kreisen Potsdam und Oranienburg keine älteren Sprecher mehr finden, die die alte mittelmärkische Mundart noch beherrschten. Eine Feststellung ..., daB die niederdeutsche Mundart des Barnim ausgestorben sei, kann nur bestätigt werden" (Wiese 1993 , S. 542). 
S. 41). ${ }^{9}$ Nachhaltige mundartliche Verfallserscheinungen gibt es auch am unteren Niederrhein (dem Kleverländischen). Hier ist der Dialekt als Muttersprache verschwunden (Macha 1993, S. 614). Ähnliches wird über das Niederdeutsche aus dem hessischen Nordwesten (zwischen Korbach und Hofgeismar) berichtet. ${ }^{10}$

Der Dialektabbau in den Randbereichen des Niederdeutschen ist nicht zu übersehen, und doch gibt es immer wieder überraschende Befunde. So haben jüngste Untersuchungen im Elbostfälischen eine nicht erwartete Dialektstabilität aufgezeigt; nicht erwartet weil es sich um eine ostfalische Dialektlandschaft handelt, die zudem jahrzehntelang einer dialektfeindlichen Kulturpolitik ausgesetzt gewesen ist. ${ }^{11}$ Erhebungen mit der gesamten erwachsenen Einwohnerschaft in 12 Dörfern nordwestlich von Magdeburg (mehr als 2.000 Personen) haben eine gute bis sehr gute aktive Dialektkompetenz bei fast $\mathbf{4 3} \%$ der Befragten erbracht, die ebenso qualifizierte passive Kompetenz beläuft sich dort auf $65 \% .^{12}$ Diese genauen Befragungen bestätigen ältere Beobachtungen aus der Magdeburger Börde, wo „der nd. Dialekt ... noch immer vorhanden ist und häufig verwendet wird, trotz der Nähe einer Großstadt ..., trotz der Nachbarschaft des md. Dialektgebietes" (Schönfeld 1991, S. 195).

Die Untersuchungen in Sachsen-Anhalt zeigen also Kompetenzwerte, die in der Nähe von denjenigen für ländliche Gemeinden im niederdeutschen Gebiet der alten Bundesrepublik liegen. Hier haben in einer Repräsentativstudie von $198466 \%$ von sich behauptet, sie sprechen gut bis sehr gut Niederdeutsch, $84 \%$ haben sich dieses Niveau im Verstehen zugesprochen. ${ }^{13}$ Als traditionell dialektstarke Regionen konnten in der erwähnten

9 Folgerichtig konzentriert sich die derzeit laufende Befragung zum Sprachgebrauch im Land Brandenburg nicht auf den Dialekt, sondern auf die Umgangssprache (Brief von Prof. Dr. J. Gessinger, Potsdam, vom 15.8.1995 an mich).

„... daB im niederdeutschen und niederhessischen Bereich Dialektgebrauch bei den Kindern fast verschwunden ist" (Dingeldein 1989, S. 55).

11 „In Mecklenburg wurde das Niederdeutsche und seine Verwendung durch die Behörden stärker gefördert, durch Medien, Presse und Rundfunk. Im Magdeburger Gebiet wurde das behindert ..., wenn sich beispielsweise Zirkel, die sich mit Niederdeutsch beschäftigten, in Schulen bildeten, dann stellten sich die Bezirksbehörden im Bezirk Magdeburg bzw. im alten Land Sachsen-Anhalt dagegen. Eine Förderung fehlte vollständig" (Helmut Schönfeld in Speckmann (1991, S. 208); (vgl. noch Föllner (1995, bes. S. 44); Bunners (1990, bes. S. 142)).

12 Föllner (Hg.) (1995); Stellmacher/Föllner (1995).

13 Stellmacher (1987, S. 29). 
Studie Schleswig-Holstein ( $47 \%$ sprechen sehr gut/gut Niederdeutsch) und das nördliche Niedersachsen (50\% sprechen sehr gut/gut Niederdeutsch) bestätigt werden. ${ }^{14}$ Für Mecklenburg-Vorpommern liegen vergleichbare Zahlen nicht vor. Hier wird eine Dialektkenntnis von 16$26 \%$ angenommen. ${ }^{15}$ Hochrechnungen kleinräumiger Befragungen in Vorpommern ergeben, dab der „Anteil derjenigen, die (das Niederdeutsche) täglich oder häufig sprechen, schwankt von vielleicht $10 \%$ in den größeren Städten, etwa 30 \% in ländlichen Gemeinden und Kleinstädten bis etwa zu $80 \%$ in wenigen Reliktgebieten" (Herrmann-Winter 1995, S. 186).

Über die Vitalität des Saterfriesischen gibt es bisher nur Schätzwerte. Dem versuche ich derzeit mit einer Befragung von $10 \%$ der in der Gemeinde Saterland lebenden erwachsenen Personen zu begegnen. Bei den bisher 751 Aufnahmen haben $82 \%$ der Befragten angegeben, niederdeutsch zu sprechen, aber nur $40 \%$ haben das auch für das Saterfriesische bestätigt. Die Nachfrage, ob sie in der zurückliegenden Woche ein Gespräch auf saterfriesisch geführt hätten, hat von den $40 \%$ knapp die Hälfte mit , ja" beantwortet.

Für das Nordfriesische sind die auffallende dialektale Zersplitterung und die geringen Sprecherzahlen kennzeichnend. Genaue Angaben fehlen, man schätzt die Zahl der aktiven Nordfriesischsprecher zwischen 6.000 und $10.000 .{ }^{16}$ Bei diesem Forschungsstand ist es willkommen, wenn punktuell genaue Zählungen vorgenommen worden sind, wie in der 600Seelen-Gemeinde Bargum in der Nordergoesharde an der Grenze zum dialektstarken Mooringer Gebiet. Gut $12 \%$ der Bargumer ist auf die Sprachkompetenz hin befragt worden. Dabei entschieden sich 12,4\% für eine gute aktive friesische Kompetenz, 79,5\% für eine solche im Niederdeutschen, 4,1\% können gut Südjütisch sprechen und 5,5\% gut Reichsdänisch. Das Hochdeutsche wird von allen gut beherrscht; wem

14 Ebda., Abb. 7. Zu den dialektstarken niederdeutschen Gebieten zählt traditionell Ostfriesland. Hier behauptet sich der Dialekt ("Regionalsprache”) auch im Berufsleben. So wird, wie eine Studie des Regionalen Pädagogischen Zentrums der Ostfriesischen Landschaft 1995 bei 495 Berufsschülern in Leer und Aurich ergeben hat, bes. in kundenorientierten Berufen (Einzelhandel) auf dem Land überwiegend niederdeutsch gesprochen. In handwerklichen Berufen ist die "Regionalsprache” die dominierende innerbetriebliche Umgangssprache.

15 Löffler (1994, S. 143).

16 Menke (1989, S. 61); Kōōp (1991, S. 76). 
diese Feststellung überflüssig erscheint, sei gesagt, dab in der Gemeinde Bohmstedt bei Breklum "einige ältere Personen (angaben), Hochdeutsch lediglich 'ein wenig' sprechen zu können" (Kööp 1991, S. 73). Besonders gefährdet scheinen die Ortsmundarten zu sein, in Bargum sprechen schon etwas mehr das ausgebaute Mooringer Frasch als den ${ }_{n}$ Basisdialekt" des Bargumer Friesisch.

Stabile Friesischgebiete sind das Ostermooringer Festlandsfriesisch und das Westföhringer Inselfriesisch. Sehr schwach vertreten ist das Nordfriesische nur noch in der Goesharde (in der Südergoesharde ist es 1980 ausgestorben) und der Karrharde sowie auf den Halligen, die heute nicht mehr zum nordfriesischen Sprachgebiet gerechnet werden können.

Für das Südjütische werden gegenwärtig "vielleicht 3.500" Sprecher angenommen (Menke 1989, S. 60). „Reinsprachig-plattdänische Dörfer” hat es südlich der Staatsgrenze schon 1937 nicht mehr gegeben (Selk 1986, S. 180). Der Rückgang des Südjütischen in der Erwachsensprache wird von P. Selk im Laufe eines halben Jahrhunderts auf ca. $50 \%$ beziffert. Den Sprachwandel stellt er so vor. „Das Hochdeutsche als die Sprache der Erwachsenen mit den Kindern bricht in das zunächst reinsprachig dänische Dorf ein; diese Sprache schlägt sozusagen eine Bresche. Die plattdeutsche Schwester folgt ihr dann, das Gebiet sprachlich endgültig eindeutschend. Das Plattdeutsche folgt aber erst dann, wenn das Hochdeutsche bereits einen gewissen Einfluß gewonnen hat" (Selk 1986, S. 179). Diese Abfolge beim Sprachwandel unterscheidet sich von der Situation in Nordfriesland. Dort sind die friesischen Ortsdialekte zuerst vom Niederdeutschen bedrängt worden. Erst ab der Mitte unseres Jahrhunderts tritt die deutsche Standardsprache als direkter Konkurrent des Friesischen auf. Das Niederdeutsche ist im intergenerationellen Sprachenwechsel jetzt keine Gefahr mehr. Beide, Friesisch und Niederdeutsch, werden zu Verlierersprachen.

Bleibt noch ein Hinweis zum Reichsdänischen in Schleswig-Holstein. Die dänische Minderheit umfaßt nach eigenen Einschätzungen ca. 50.000 Menschen, die als Sprecher dieser Sprache in Frage kommen. Das Verhältnis zum Südjütischen ist locker, obwohl das Reichsdänische bestrebt ist, den historischen Anspruch der dänischen Volkssprache in Schleswig fortzusetzen. Die Plattdänischsprecher sehen im Reichsdänischen "nicht unbedingt ihre zugehörige Dach- und Hochsprache ... (mögliche Gründe: linguistischer Abstand, landfremde Sprache, geschichtliche Sonderstellung des alten Herzogtumis)" (Menke 1989, S. 60$) \cdot{ }^{17}$

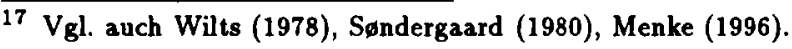


Von linguistischer Seite wird hervorgehoben, daB die zahlreichen Einmischungen aus dem Hoch- und dem Niederdeutschen das Südjütische zu einer "hybridisierten Varietät" haben werden lassen ${ }^{18}$, was für den Weiterbestand dieser Sprache nicht ungefährlich ist.

Und schlieblich die mitteldeutsche Sprachinsel in Harz. In den niedersächsischen Bergstädten Clausthal-Zellerfeld, Altenau, Wildemann, Lautenthal und Sankt Andreasberg (alle im Kreis Goslar) " gibt es zwar noch eine ganze Menge Leute, die die Sprache in Wort und Schrift verstehen, aber nur noch wenige, die sie sprechen, und ... noch weniger, die sie auch schreiben können. Zwar hat es immer wieder Bemühungen gegeben, diesen Verfall aufzuhalten, aber das zeigte stets nur vorübergehend Wirkung" (Astheimer 1993, S. 235). ${ }^{19}$

\section{Das Mehrsprachigkeitsverhältnis}

$\mathrm{Zu}$ den qualitativen Merkmalen einer Sprachsituation wird das gleichwertige bzw. ungleichwertige Verhältnis zwischen den beteiligten Sprachen gezählt. Dabei ist Gleichwertigkeit der Ausnahmefall. Schon der Umstand, dab sich eine Sprache expansiv, eine andere regressiv verhält, hat Funktionsverschiebungen zur Folge, die mit der Formel vom Sprachausbau und Sprachabbau erfaBt werden.

Das Niederdeutsche und das (Nord-)Friesische verzeichnen einen nicht auf die Nahbereiche beschränkten Verwendungsumfang. Er erstreckt sich quer über die Domänen des kommunikativen Nah- und Fernbereichs. Deshalb paBt zur norddeutschen Sprachsituation auch nicht das Diglossie-Konzept. An einigen niederdeutschen Texten (siehe Anlage) möchte ich das belegen.

Die zehn Textbeispiele lassen sich sechs Kommunikationsbereichen zurechnen, den Nahbereichen I und II sowie den Fernbereichen III, IV, V und VI.

Im einzelnen ergibt das diese Zuordnung:

I. Briefliche Benachrichtigung

Text 1, 2: Rundbrief, Vereinsnachricht

18 Dyhr (1990).

19 Im 18. Jahrhundert sind in Pommern (bei Pasewalk und Ueckermūnde) und in Ostfalen (bei Braunschweig) westmitteldeutsche Kolonien entstanden, dazu Herrmann-Winter (1995, S. 183) und Karch (1978). 
II. Büropraxis

Text 3: Gebrauchstext, hier formalisierte Kurzmitteilung für die Büropraxis

III. Öffentliche Medien gedruckt: Text 4, 5: Zeitschrift (Editorial), Tageszeitung (Kolumne)

IV. Öffentliche Medien

elektronisch Text 6: Rundfunknachricht

V. Sachprosa

Text 7: wissenschaftlicher Text - Sprachwissenschaft

(Dissertationsresümee)

Text 8: wissenschaftlicher Text - Theologie

Text 9: Verfassungstext (nichtamtliche Fassung)

VI. Öffentliche Rede

Text 10: Parlamentsdebattenbeitrag

Mit diesen Verwendungen erfüllt das Niederdeutsche Ausbaukriterien, wie sie H. Kloss für die Entwicklung einer "Sprachvariante ... zur selbständigen Einzelsprache" (Kloss 1987, S. 304) vorgegeben hat. Die Produzenten der Ausbautexte sind alles auch kompetente Standardsprecher. Sie erlauben sich die "Referenz auf ein zweites Sprachsystem, in dem (sie) inhaltliche und funktionale Eigenschaften besonderer Qualität (vermuten). Neben komischen Wirkungen (vgl. Text 10, D. St) wird dem ... Gebrauch (des Niederdeutschen) ... - im Sinne eines metaphorical switching - eine die soziale Situation der Sprecher verändernde Funktion unterlegt" (Kontaktaufnahme, Vertrautheit, Solidarisierung, Unterhaltung) (Diercks 1994, S. 232)). Es darf aber niemals übersehen werden, daB das Niederdeutsche seinen Verwendungsschwerpunkt nicht in den Ausbaudomänen, sondern im Reden über Persönliches und Alltägliches hat.

Deshalb kritisiert z.B. der im Kieler Landtag verabschiedete Landesplan Niederdeutsch, „daB dem Niederdeutschen in den Medien überwiegend eine Rolle zugewiesen wird, mit der das Niederdeutsche von den ernsteren Themen der Politik, der Berufswelt, der Bildung, der Geschichte und der Gesellschaft ausgeschlossen wird" (Schleswig-Holsteinischer Landtag. Landesplan Niederdeutsch, vorgelegt am 14.6.1993, beschlossen am 1.7.1994).

Die Texte in der Anlage stehen für ein code-switching als Ausdruck einer nicht domänenbegrenzten Zweisprachigkeit. Anders verhält es sich beim sog. code-mixing, das als alternierendes Sprechen nicht selten bei Norddeutschen $\mathrm{zu}$ beobachten ist, die dabei keine bestimmte Funktionsabsicht verfolgen und ihre Sprache nicht auszubauen beabsichtigen, z.B. 
„Wi kamen hen, un de Karren is nich dor, einfach nicht mehr da! Und das hatte der Dicke getan. - Hei hett den Karren einfach nah dei anner Städ' weghaalt un hett uns nicks seggt. Er hat uns gar nichts gesagt. Und wenn er ihn wegnimmt, muß er ihn auch wiederbringen. - Hei möt em tau'n wenigsten wedder henstellen dor, wur hei em wegnahmen hett. Sonn' Aas! Un wi koenen säuken. - Aber der soll uns mal wiederkommen, denn kann hei wat biläben" (Dahl 1974, S. 386f.).

Die norddeutsche Sprachsituation ist in Gänze mit den Begriffen Bilingualismus und Diglossie nicht zu erfassen. Bilingual verhält sich der um Sprachausbau Bemühte nur scheinbar, was das häufig anzutreffende Verfahren des metaphorical switching als Diglossiemerkmal beweist (vgl. Anrede und GruB in Text 1 und die Anrede in Text 10). Ungeachtet der Ausbautexte ist festzuhalten, $\mathrm{daB}$ das Niederdeutsche nirgends mehr einziges Verständigungsmittel ist, obwohl genau das die sicherste Gewähr für eine Fortexistenz der Sprache wäre. ${ }^{20}$ Doch auch eine begrenzt eingesetzte Sprache kann überleben, wenn die Sprachgemeinschaft in ihr die Identifikationssprache (gegenüber einer "fremden" Funktionssprache) sieht, wie das zum Teil beim Friesischen der Fall ist. Um das zu prüfen, ist ein Blick auf das kulturelle Umfeld nötig.

\section{Das (kulturelle) Umfeld}

Hierbei handelt es sich um das „institutionelle Netzwerk aus Vereinen und Verbänden, Bühnen und Verlagen. Literaten-, Pastoren- und Lehrergruppen ..., das fast den ganzen Norden überzieht. Allerlei spartenspezifische Publikationsorgane, Tagungen, Gremien usw. gehören ebenfalls dazu, kurzum, die Branche verfügt über eine ziemlich feste organisatorische Struktur" (Schuppenhauer 1994, S. 7). Die "Branche” ist Ergebnis von Bemühungen um die Rettung des Niederdeutschen, die seit der Rezeption des Hochdeutschen in der sog. Übergangszeit (16./17. Jahrhundert) einsetzen und in zu- und abnehmender Intensität bis heute andauern. Dabei ist dem "Pro"-Niederdeutsch von Beginn an ein "Contra" entgegengehalten worden, so daB sich die jeweiligen Positionen in zwei Kommentarsträngen bündeln: „Der eine bezieht sich auf die Durchsetzung des (Hochdeutschen), von seiner kommunikativen Reichweite und seiner kognitiven Leistungsfähigkeit her argumentierend; der andere, vor dem Hintergrund der regional-kulturellen Identität, eröffnet das Thema der 'Modersprak' mit reicher Konsequenz" (Möhn 1988, S. 72). Da heiBt es auf der einen Seite bedauernd, dab die "Heldensprache der Cimbern und Sachsen" "templisque ac curiis, imo ipsis pene domibus exulat", so

Dazu Peeters (1991). 
1691 aus dem Schleswigschen überliefert (Raupach 1984, S. 74f.). Auf der anderen Seite wird $50 \mathrm{~J}$ ahre später (1743) von einem Oldenburger Anonymus empfohlen, , dab es nützlich und möglich sey, die niedersächsische Sprache allmählich gar abzuschaffen" (Schulte Kemminghausen 1939, S. 63). Und ob Goethes Einschätzung des Niederdeutschen als ${ }_{n}$ sanftes, behagliches Urdeutsch" in einer Rezension von Johann Heinrich VoB' "Lyrischen Gedichten" (1804) ${ }^{21}$ ein Pro- oder Contra-Kommentar ist, hätte eine genauere Prüfung erst noch zu erweisen.

Mit dem Wiedererstarken der mundartlichen Literatur ab der Mitte des vorigen Jahrhunderts erhält auch die niederdeutsche Bewegung Auftrieb und EinfluB. Der niederdeutsche Buchmarkt bietet z.B. 1991 mehr als 1.700 Titel an. ${ }^{22}$ Auch die Schöpfung einer originellen Sprachform ist der breit entwickelten niederdeutschen Literatur zuzuschreiben: das Missingsch, das weniger natürliche Sprechsprache ist als eine Art von Stadtliteratur, vgl. Walter A. Kreyes Bremen-Buch "Was'n in Bremen so sacht un wo ein fein auf hören muf" (1973).

Ohne die niederdeutsche Bewegung nicht denkbar sind die zahlreichen Vereinsgründungen, die im letzten Drittel des 19. Jahrhunderts einsetzen und bis heute andauern und für die Aufwertung des Niederdeutschen viel getan haben (und tun), z.B. der Plattdeutsche Centralverein (1867 in Berlin gegründet), der Verein für niederdeutsche Sprachforschung (1874 in Hamburg gegründet), die Dramatische Gesellschaft (heute Ohnsorg-Theater) (1902 in Hamburg gegründet), die Freie Vereinigung von Freunden der niederdeutschen Sprache und Literatur (heute Quickborn-Vereinigung) (1904 in Hamburg gegründet), die Fehrs-Gilde (1916 in Hamburg gegründet), der Arbeitskreis für niederdeutsche Sprache und Dichtung "Bevensen-Tagung" (1948 in Bevensen gegründet), das Institut für niederdeutsche Sprache e.V. (1972 in Bremen gegründet), das Ostfälische Institut der DEUREGIO Ostfalen e.V. (1994 in Ummendorf/Bördekreis gegründet). ${ }^{23}$ Auch die Dänischgesinnten in Schleswig haben sich in Vereinigungen, sog. Vortragsvereinen, die Strukturen für eine politische Interessenvertretung geschaffen.

21 Goethe, Johann Wolfgang (1804, S. 278).

22 Plattdeutsch im Buchhandel (PiB), hg. vom Institut für niederdeutsche Sprache. Bremen 6. Auflage 1991.

23 Dazu Möhn (1978, bes. S. 8); Institut für niederdeutsche Sprache. 19721982, hg. vom Vorstand des Institut für niederdeutsche Sprache. Bremen 1982. Zur friesischen Bewegung und ihren Vereinsgrūndungen vgl. Steensen (1986). 
Heute gibt es in Norddeutschland 16 Heimatbünde und Landschaftsverbände mit vielfältigen niederdeutschen Einrichtungen. Wie viele niederdeutsche Vereine es genau sind, vermochte selbst das wohlinformierte Bremer Institut für niederdeutsche Sprache nicht zu sagen. Niederdeutsche Theatergruppen soll es mehrere Tausend geben.

Die Zahl der Pastoren, die Niederdeutsch in ihrem Dienst verwenden, wird auf 700 geschätzt. Wie mächtig die Branche insgessmt ist, beweist ihr Einsatz für die Aufnahme des Niederdeutschen in die Europäische Charta der Regional- und Minderheitensprachen. ${ }^{24}$ Von dem schlichten Appell (" Proot doch Platt, schodt di dat?!") oder dem Stempelaufdruck bei der ausgehenden Post der schleswig-holsteinischen Ministerpräsidentin ( Art. 1 Grundgesetz) bis zum wirkungsmächtigen Lobbyismus erstrecken sich die Aktionen des niederdeutschen (kulturellen) Umfelds. Erfolgreicher Ausdruck dessen ist die Aufnahme der Pflege des Niederdeutschen in die Landesverfassung von Mecklenburg-Vorpommern (Artikel 16,2). Der Unterstützung des Friesischen als Identitätsmerkmal der friesischen Volksgruppe in Schleswig-Holstein dient der Artikel 5 der Landesverfassung (in der Fassung vom 13.6.1990); hier erfährt auch die dänische Minderheit verfassungsrechtlichen Schutz.

Aus dem (kulturellen) Umfeld wird den Zuständigen und denen, die sich für zuständig erklären, immer wieder die Frage gestellt, wie lange es das Niederdeutsche noch geben werde. Die Frage ist Ausdruck einer als existentiell empfundenen Bedrohung der Sprache und wohl auch eines Unterlegenheitsgefühls (gegenüber der Standardsprache). Ein Zeichen dafür sind die verbreiteten Humorpostulate des Niederdeutschen und das häufig begegnende metaphorical switching. ${ }^{25}$ Antworten auf die Frage nach dem Weiterleben des Niederdeutschen fallen nicht selten wohltuend nüchtern aus und sind Ergebnis einer Distanz erkennenlassenden Sprachreflexion. So auch die Reaktion des kalenbergischen Autors Konrad Tegtmeier auf eine solche Frage: „Un wenn de plattduitsche Sprake noch genaug Kraft in'er Wörtel hett und (sic!) wenn se bleben sall, denn blifft se ok. Et is gar nich so lichte, wat doot tau kregen, un wenn se eben doch verdammet is, unnertaugahn, denn is et eben vorbee, denn kann'n da ok nich viel giegen maken" (Gespräche 1964, S. 122).

Wenn die niederdeutsche (und jede andere norddeutsche) Sprachform zum Untergang verdammt ist, dann ist nichts dagegen zu machen. So

24 Speckmann (Hg.) (1991, S. 5); Wirrer (1993); Menge (1995).

25 Zum regionenspezifischen Humor siehe Schröder (1995). 
oder ähnlich wird es auch der Wissenschaftler sagen. Doch den Stand einer Sprache genau und umfassend festzulegen, bleibt seine Aufgabe. Eine Sprache gleich welchen Status wird nur dann überleben, wenn die Sprachgemeinschaft in ihr mehr als ein instrumentell-kommunikatives Mittel sieht. In dieser Hinsicht vermag das (kulturelle) Umfeld einiges zu bewegen, wie an vielen Beispielen aus unseren Tagen zu sehen ist. Die Wissenschaft wird also das Umfeld mit der gleichen Sorgfalt zu beobachten haben wie die sprachlichen Entwicklungen. Sie muB mögliche Übertreibungen, z.B. den Versuch einer "Ethnisierung” von Sprachformen, erkennen und davor warnen, denn wie der Volkskundler Konrad Köstlin zurecht feststellt, hat sich Sprache als Mittel der Ausgrenzung immer wieder tauglich machen lassen. ${ }^{26}$

\section{Zusammenfassung}

Die norddeutsche Sprachsituation ist durch Mehrsprachigkeit gekennzeichnet. Daran beteiligt sind neben der dominierenden deutschen Standardsprache das Niederdeutsche, Friesische und Dänische. Das Verhältnis dieser Sprachen zueinander wird durch ein diffuses sprachgeschichtliches Wissen ("Mythos") und offenbare Substanzunterschiede ("Abstand”) außerordentlich kompliziert und ist mit den geläufigen Mehrsprachigkeitskennzeichnungen (Bilingualismus, Diglossie) nur unzureichend zu beschreiben. Da die Abstandsprachen Sprachen sind, die „väle Mundorden" haben (siehe das einleitende Zitat), ergibt sich sozusagen eine Diglossiesituation zweiter Ordnung. ${ }^{27}$ Damit erweist sich die Sprachsituation in Norddeutschland als etwas Besonderes und rechtfertigt die Vorstellung auf einer IdS-Tagung zu den „Varietäten des Deutschen”.

\section{Literatur}

Astheimer, Kurt (1993): Sprachinsel Oberharz. In: Niedersachsen 93, S. 235237.

Blume, Herbert (1980): Zur funktionalen Konkurrenz von Ostfalisch, Nordniedersāchsisch und Hochdeutsch im sūdlichen Ostfalen. In: ZGL 8, S. 314327.

Bunners, Christian (1990): Die heutige Verwendung des Niederdeutschen in Kirchengemeinden in der DDR. In: Buske, N. (Hg.) (1990): Niederdeutsche Bibeltradition. Entwicklung und Gebrauch des Niederdeutschen in der Kirche. Berlin und Altenburg. S. 139-153.

Dahl, Eva-Sophie (1974): Interferenz und Alternanz - zwei Typen der Sprachschichtenmischung im Norden der Deutschen Demokratischen Republik.

${ }^{26}$ Köstlin (1995); auch Michelsen (1993, bes. S. 135ff.).

${ }^{27}$ Einen Einblick in diese Fragestellung gibt Blume (1980). 
In: Aktuelle Probleme der Sprachlichen Kommunikation. Soziolinguistische Studien zur sprachlichen Situation in der Deutschen Demokratischen Republik. Berlin. S. 339-387.

Diercks, Willy (1994): Niederdeutsch in der Stadt Schleswig. Zu Attitūden und zur Sprachverwendung. Stuttgart. (ZDL-Beiheft 86).

Dingeldein, Heinrich J. (1989): Das Mittelhessische. In: Hessisches. H. Friebertshāuser zum 60. Geburtstag am 21. Mārz 1989. Marburg. S. 9-69.

Dyhr, Mogens (1990): Hybridisiertes Südjütisch. In: Kremer, L./Niebaum, H. (Hg.) (1990): Grenzdialekte. Studien zur Entwicklung kontinentalwestgermanischer Dialektkontinua. Hildesheim und Zūrich. S. 25-47.

Feitsma, Anthonia (1995): Taal, minderheidstaal en dialekt. In: Cajot, J./Kremer, L./Niebaum, H. (Hg.) (1995): Lingua Theodisca. Festschrift für J. Goossens. Münster/Hamburg. S. 885-891.

Föllner, Ursula (1995): Niederdeutsch in Sachsen-Anhalt. In: Niederdeutsches Korrespondenzblatt 102, S. 44-49.

Föllner, Ursula (Hg.) (1995): Untersuchungen zum Niederdeutschen in Sachsen-Anhalt. Erste Ergebnisse eines Forschungsprojektes der Arbeitsstelle Niederdeutsch an der Otto-von-Guericke-Universitāt in Magdeburg. Oschersleben. (Otto-von-Guericke-Universitāt in Magdeburg. Schriftenreihe der Arbeitsstelle Niederdeutsch 1).

Gespräche (1964): Gespräche mit plattdeutschen Autoren. Hg. von den Mitarbeitern des Niedersächsischen Wörterbuchs. Neumünster.

Goethe, Johann Wolfgang (1804): Lyrische Gedichte von Johann Heinrich VoB. In: Jenaische Allgemeine Literaturzeitung Nr. 91, 92 vom 16., 17.4.1804. In: Weimarer Ausgabe (Sophienausgabe) Teil 1, Band 40. Weimar (1901). S. 263-283.

Hansen, Reimer/Johannesen, Peter Ivar/Runge, Johann/Steensen, Thomas (1993): Minderheiten im deutsch-dänischen Grenzbereich. Landeszentrale für politische Bildung Schleswig-Holstein. Kiel. (Gegenwartsfragen 69).

Herrmann-Winter, Renate (1989): Frau Apotheker kaufte ihren Hut hochdeutsch. Prominente über Platt. Rostock.

Herrmann-Winter, Renate (1995): Sprache und Sprechen in Pommern. In: Niederdeutsches Jahrbuch 118, S. 165-187.

Hoppenbrouwers, C. (1990): Het Regiolect van dialect tot Algemeen Nederlands. Muiderberg.

Institut fūr niederdeutsche Sprache (1982). Hg. vom Vorstand des Instituts für niederdeutsche Sprache. Bremen.

Karch, Dieter (1978): Braunschweig-Veltenhof. Pfälzische Sprachinsel im Ostfälischen. Tübingen. (Phonai 11).

Kettner, Bernd-Ulrich (1988): Die norddeutsche Umgangssprache - eine neue Zweitsprache? In: Norddeutsch und Zweisprachigkeit. Befunde - Vergleiche - Ausblicke. Leer. S. 95-113.

Kloss, Heinz (1987): Abstandsprache und Ausbausprache. In: Ammon, U./Dittmar, N./Mattheier, K.J. (Hg.) (1987): Soziolinguistics - Soziolinguistik. Berlin und New York. S. 302-308. (HSK-Band 3.1.). 
Kööp, Karl-Peter (1991): Sprachentwicklung und Sprachsituation in der Nordergoesharde. Von der Mitte des 19. Jahrhunderts bis zur Gegenwart. Bräist/Bredstedt.

Köstlin, Konrad (1995): Regionale Sprachen - Die Moderne als Deutung (Vortragsresūmee). In: Niederdeutsches Korrespondenzblatt 102, S. 41.

Löffler, Heinrich (1994): Germanistische Soziolinguistik. 2. Auflage. Berlin.

Macha, Jürgen (1993): „Wie die alten sungen ...?” Generation und Sprache im Rheinland. In: Mattheier, K.J. u.a. (Hg.) (1993): Vielfalt des Deutschen. Festschrift für W. Besch. Frankfurt/M. u.a. S. 601-618.

Menge, Heinz H. (1995): Rehabilitierung des Niederdeutschen. Erwartungen an die europäische Sprachenpolitik. In: ZGL 23, S. 33-52.

Menke, Hubertus (1989): Dialekte im Grenzraum. In: Bichel, I./Herold, D./Holm, V. (Hg.) (1989): Niederdeutsch im Unterricht an Gymnasien. Anregungen und Arbeitshilfen. Kiel. S. 60-63.

Menke, Hubertus (1996): 'Ich bin ein Dāne und spreche deutsch'. Zur Sprachgeschichte und Sprachenpolitik im deutsch-dänischen Grenzraum. In: Marti, Roland ( $\mathrm{Hg}$.) (1996): Sprachenpolitik in Grenzregionen. Politique linguistique dans les régions frontalières. Language Policy in Border Regions. Polityka językowa na pograniczach. Saarbrücken. S. 137-161.

Michelsen, Friedrich W. (1993): Bilden die niederdeutschen Mundarten eine soziokulturelle Einheit? Anmerkungen zu einer aktuellen Frage. In: Niederdeutsches Wort 33, S. 125-137.

Mōhn, Dieter (1978): Niederdeutsches Sprachleben in Hamburg wāhrend der letzten hundert Jahre. In: Quickborn 68, S. 2-15.

Mōhn, Dieter (1988): Kommentare zur Zweisprachigkeit in der Geschichte des Niederdeutschen. In: Norddeutsch und Zweisprachigkeit. Befunde - Vergleiche - Ausblicke. Leer. S. 71-83.

Peeters, Yvo J.D. (1991): Verfremdende Folgen einer allgemeinen Zweisprachigkeit für sprachliche Minderheiten. In: Quickborn 81, S. 124-128.

Raupach, Bernhard (1984): De Linguae Saxoniae inferioris neglectu atque contemtu injusto. Von unbilliger Verachtung der plat-Teutschen Sprache (1704). Übersetzt von Sievert Graf Wedel. Bearbeitet von Wolfgang Lindow. Leer.

Scharnhorst, Jūrgen (1978): Die Sprachsituation in der Deutschen Demokratischen Republik. In: Sprachpflege 27, S. 1-6.

Schōnfeld, Helmut (1986): Prozesse bei der Herausbildung regionaler Umgangssprachen im 19. und 20. Jahrhundert (am Beispiel der berlinischbrandenburgischen Umgangssprache). In: Lösch, W. (Hg.) (1986): Umgangssprachen und Dialekte in der DDR. Jena. S. 162-175.

Schōnfeld, Helmut (1991): Die niederdeutsche Sprache in den Lāndern SachsenAnhalt und Brandenburg. In: Niederdeutsches Jahrbuch 114, S. 175-201.

Schröder, Martin (1995): „Derbe, aber bieder.” Überlegungen zur Genese von Ethno- und Sprachstereotypen innerhalb des niederdeutschen Humordiskurses. In: Niederdeutsches Jahrbuch 118, S. 189-210. 
Schulte Kemminghausen, Karl (1939): Mundart und Hochsprache in Norddeutschland. Neumünster.

Schuppenhauer, Claus (1994): Plattdeutsch als Auftrag. Das Institut für niederdeutsche Sprache. Versuch einer Zwischenbilanz. Leer.

Selk, Paul (1986): Die sprachlichen Verhältnisse im deutsch-dänischen Sprachgebiet südlich der Grenze. Eine statistisch-geographische Untersuchung. Hamburg (Korrigierter Nachdruck der Ausgaben von 1937 und 1940).

Semenjuk, N.N. (1985): Soziokulturelle Voraussetzungen des Neuhochdeutschen. In: Besch, W./Reichmann, O./Sonderegger, S. (Hg.) (1985): Sprachgeschichte. Ein Handbuch zur Geschichte der deutschen Sprache und ihrer Erforschung. Berlin und New York. S. 1448-1466. (HSK - Band 2.2).

Søndergaard, Bent (1980): Vom Sprachenkampf zur sprachlichen Koexistenz im deutsch-dānischen Grenzraum. In: Nelde, P.H. (Hg.) (1980): Sprachkontakt und Sprachkonflikt. Wiesbaden. S. 297-305. (ZDL-Beiheft 32).

Speckmann, Rolf ( $\mathrm{Hg}$.) (1991): Niederdeutsch morgen. Perspektiven in Europa. Beitrāge zum Kongreß des Instituts für niederdeutsche Sprache. Lüneburg 19.-21.10.1990. Leer.

Steensen, Thomas (1986): Die friesische Bewegung in Nordfriesland im 19. und 20. Jahrhundert (1879-1945). Neumünster.

Stellmacher, Dieter (1987): Wer spricht Platt? Zur Lage des Niederdeutschen heute. Eine kurzgefabte Bestandsaufnahme. Leer.

Stellmacher, Dieter/Föllner, Ursula (1995): Die Mundarten in der DEUREGIO Ostfalen. Verbreitung, Wandel, Gebrauch. Mannheim. (Veröffentlichungen des Ostfälischen Instituts der DEUREGIO Ostfalen, Band 1).

Vinogradov, V.A. (1990): Jazykovaja situacija. In: Jarceva, V.N. (Hg.) (1990): Lingvistiðeskij enciklopediðeskij slovar. Moskva. S. $616 \mathrm{f}$.

Wiese, Joachim (1993): Zu Problemen des Verhăltnisses von Mundart und Umgangssprache in einem Dialektwörterbuch. Dargestellt am BrandenburgBerlinisches Wörterbuch (BBW). In: Viereck, W. (Hg.) (1993): Verhandlungen des Internationalen Dialektologenkongresses. Bamberg 29.7.4.8.1990. Band 2. Stuttgart. S. 542-552. (ZDL-Beiheft 75).

Wiese, Joachim (1993): Forschungen zur Sprachgeschichte im Land Brandenburg. In: Jahrbuch für brandenburgische Landeskunde 44, S. 34-42.

Wilts, Ommo (1978): Nordfriesisch, Hoch- und Niederdeutsch in SchleswigHolstein. Zur Dynamik sprachlicher Entwicklungen in einem Mehrsprachenland. In: Ureland, P.S. (Hg.) (1978): Sprachkontakte im Nordseegebiet. Akten des 1. Symposions über Sprachkontakte in Europa. Mannheim 1977. Tübingen. S. 149-166.

Wirrer, Jan (1993): Die Europäische Charta der Regional- und Minderheitensprachen und das Niederdeutsche. In: Quickborn 83, S. 29-41. 
Anlage:

\section{- Textbeispiele -}

1. Rundbrief (oldenburgische Sprachform und Standard)

Leeve Lü!

Wie auf unserer letzten Sitzung in Soltau besprochen, soll am ... Ich bitte um baldige Rücksendung der anliegenden Karte.

Dat goh Jo good!

(Brief des Vorsitzenden der FG für hoch- und niederdeutsche Sprache des Niedersächsischen Heimatbundes vom 19.05.1988).

2. Vereinsnachricht (ostfälische Sprachform)

Inloadunge

Leeibe Fruinde, weei kumet an 19. August 1995 in Obernfeld in Eichsfelle teheope in Hotel Gasthof "Museumskrug” Kirchgasse 1. Vorrhäre drāpe weei üsch Klocke niegen in Gieboldehausen. Up dā Parkstie'e an Slotte kōnnt weei dä Wogens afstellen. (aus der Einladung zu einer Veranstaltung der Kommission für Plattdeutsche Sprache und Literatur der Arbeitsgemeinschaft Südniedersāchsischer Heimatfreunde e.V. vom 1.8.1995).

3. Gebrauchstext (ostfriesische Sprachform)

\section{Telefax}

Dat is för

Antahl/Antall Sieden (mit dit Bladd)

Utkummst gifft Heer/Froo

Telefondörwahl 
4. Editorial (nordniedersächsische Sprachform)

De Koopmannsdeel in dat Museum für Hamburgische Geschichte weer bit up den letzten Platz besett, as wi an 12. Januar Fiete Michelsen den Quickborn-Pries övergeven heff. Von Harten dankt wi den Huusherrn, Prof. Dr. Jōrgen Bracker. In dit schōne Museum is de Quickborn ja al foken to Gast ween, un uns hett dat dor jūmmers goot gefullen.

(Quickborn. Zeitschrift für plattdeutsche Sprache und Literatur. 85. Jg., Heft 1, 1995).

5. Zeitungskolumne (hamburgisches Niederdeutsch)

Clara Kramer vertellt

Dat frische Oof lacht uns allentalben an. Dat Woter löpt mi in'n Mund tosomen, wenn 'k öber den Markt gooh.

Nu twee Rezepten, de so wunnerboor in disse scheune Sommertiet paBt.

Himbeercrem - Suurkirschcrem ...

(aus "Hamburger Abendblatt" vom 29.7.1995).

\section{Rundfunknachricht (Medienplatt)}

\section{Bosnien-Ploon}

De Weltsekerheitsroot het de bosnischen Kriegsparteien opföddert, glieks wieder to verhanneln. Dat, wo se in Genf över enig worrn sūnd, schall so fastkloppt warrn. De Ploon süht so ut, dat de Moslems un Kroaten 51 un de Serben 49 Perzent vun Bosnien kriegt. De bosnische Serbenscheff Radovan Karadzic meent, dat no Genf dat Door för den Freden nu open steiht.

(Hamburg-Welle, Nachrichten vom 9.9.1995, 8.30 Uhr).

7. Wissenschaftlicher Text (nordniedersächsische Sprachform)

Liekers dat Hochdüütsche en groten Influß op dat Plattdüütsche hett, sünd de mehrsten Wöōr in mien Ünnersōken doch noch platt. Man en wichtigen Ünnerscheed is in de Spraak von de ölleren und (sic!) de jüngeren Lüüd fasttostellen: bruukt de öleren Buern doch noch recht oft ole plattdüütsche Utdrück, so ward von de jungen Lüüd lever Wōōr bruukt, de nich veel anners oder jūst so sünd as de hochdūütschen Utdrūck. 
Op lange Sicht ward dorüm de Spraak von de Wilster-MaschBuern jümmers neger an dat Hochdūūtsche ranrūcken, ok wenn dat ene oder annere Woort plattdūītsch blieven ward.

(J. Ruge, Der landwirtschaftliche Fachwortschatz in der Wilstermarsch. Generationsspezifische Untersuchungen zu seiner Entwicklung. Hamburg 1995, S. 366).

\section{Wissenschaftlicher Text (nordniedersächsische Sprachform)}

Von 1525 an, as Joachim Slüter (ca. 1490-1532) in Rostock dat ierste plattdüütsche Gesangbook (54 Leeder) bi Ludwig Dietz drucken leet, bet 1989, as in de Evangelische Verlagsanstalt Berlin/DDR dat Plattdüūtsch Gesangbauk for Meckelunborg un Vörpommern (72 Leeder) rutkaamen dä, hett dat een lange Reeg Leederbööker för Kark un Huus geven. Alleen J. Slūter sien tweete Book von 1531 (114 Leeder) is bet 1564 sōBteihnmol opleggt worden; dat nee'e Rostocker Gesangbook von 1577 (214 Leeder mit Noten) kōōm bet 1651 gor op 24 Oplaagen.

(Nachwort zu „Dor kummt een Schipp.” Plattdūūtsch Gesangbook. Hermannsburg 1991, S. 380).

\section{Verfassungstext (mecklenburgische Sprachform)}

(1) De Börgers un Bōrgerinnen von Mäkelborg-Vörpommern bekennen sick tau de Minschenrechte as den Grund von de staatliche Gemeinschaft, von Fräden un Gerechtigkeit.

(2) Dat Land Mäkelborg-Vörpommern is fōr de Minschen dor un möt so all' dei Minschen, dei in em läben oder sick ok blot in em uphollen, in ehr Wiert un Anseihn achten un in Schutz nāhmen.

(3) De in dat Grundgesetz für die Bunnesrepublik Düütschland fastleggten Grundrechte un dei Rechte, dei ein as'n düütschen Staatsbörger hett, dei sünd ein tauhūrig Deil von disse Verfatung un sei gellen as Recht genau so in Mäkelborg-Vōrpommern.

(Vörlöpige Verfatung von dat Land Mākelborg-Vörpommern, Artikel 5, hg. vom Landtag Mecklenburg-Vorpommern, Referat Presse- und Ōffentlichkeitsarbeit. Schwerin). 
10. Parlamentsdebatte (nordniedersächsische Sprachform und Standard)

J. (FDP):

Frau Präsidentin, darf ich hier Platt snacken?

Vizeprāsidentin G.:

Sie drof dat.

\section{(Beifall)}

J. (FDP):

Fro Prāsidentin, Fronslūe un Kirls hier in'n Plenorsal un alln's wat uppe Tribüne tohopelopen is! Düsse Punkt awer denn wi nu snackt, befaht sick mit dat platte Land. In väle Dörper wat blos noch platt snackt un in de lütschen Städte künnt de meisten Lūe platt verstahn. Ower 60 Perzent in Norddütschland un in Neddersassen könnt platt verstahn, dat secht de Mikrozensuserhebung. Se kūnnt ower uk hochdütsch, un wer twee Spraken spräken kann, is keen Dōskopp, glöwt dat man ...

Vizepräsidentin G.:

Herr J., Sie müßten einmal ein bißchen fixer schnacken!

$$
\text { (Heiterkeit.) }
$$

Die Redezeit ist abgelaufen.

J. (FDP):

Ick mut jo sergen, ick hebb mir (sic!) dor Gedanken awer makt, de wird jo uk fördert düsse Projekte. Interessant wör jo to wāten, woveel dat sünd un woveel fördert wurn is upp'n Lanne, wenn'n dat moll verglickt mit den Kulturverdrag von Hannober. Dat is sicher een groden Ünnerscheed. (Beifall bei der FDP und der CDU) ...

(aus Protokoll des Niedersāchsischen Landtags, 12. Wahlperiode, 84. Plenarsitzung am 10.6.1993). 


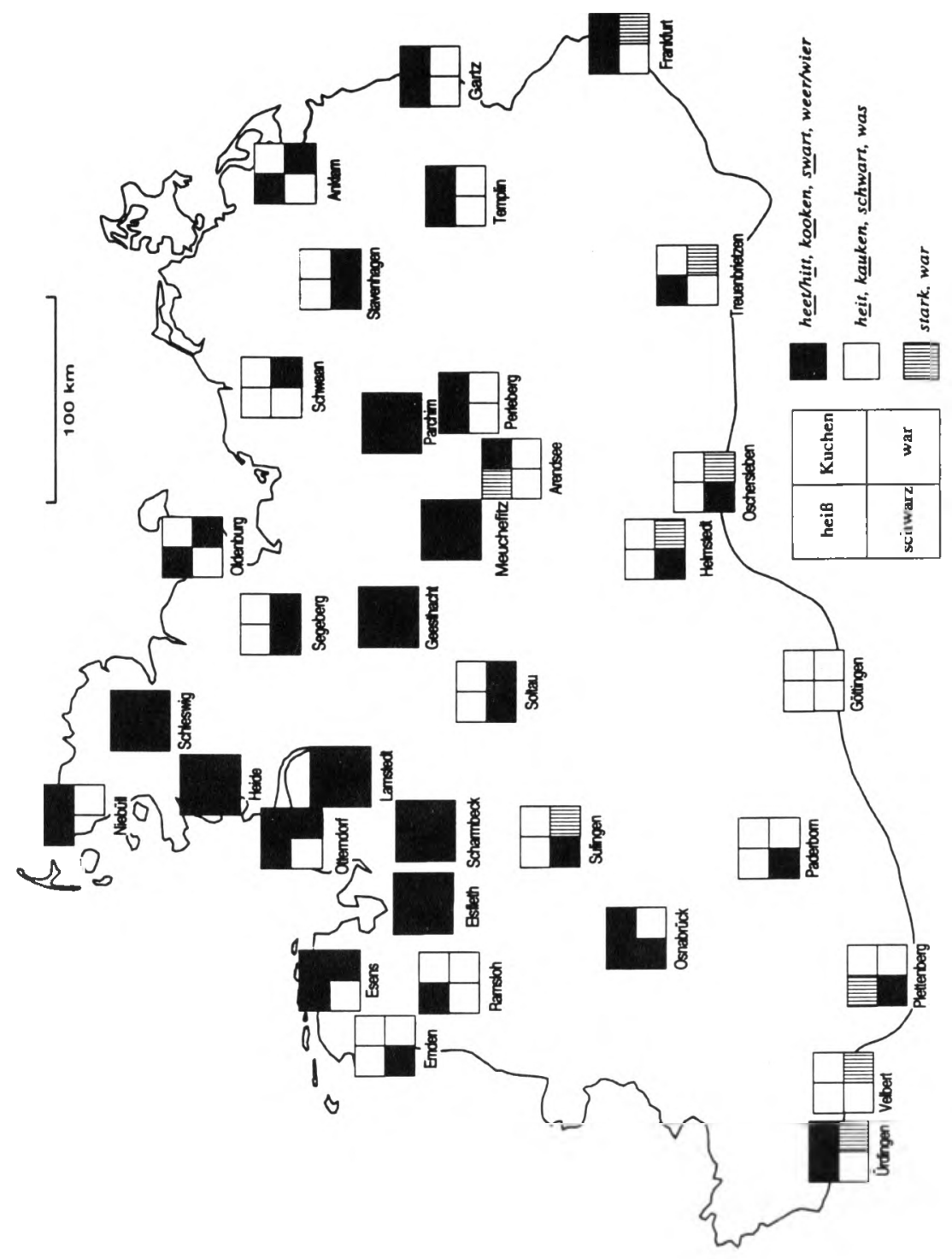

